\title{
Geometric Modeling and EM Analysis of Electrical Windings for Pulsed Alternators
}

\author{
G.A. Wedeking \\ Center for Electromechanics \\ The University of Texas at Austin \\ Austin, TX USA
}

\begin{abstract}
Pulsed alternators are state-of-the-art machines that require analytical design tools with a high level of fidelity. To meet the design needs, accurate modeling and analysis methods of the electrical windings of the machines have had to be developed. This paper will present a technique for creating accurate geometric models of racetrack style electrical windings, as well as a means of using these models to perform electromagnetic load analysis of the windings. This will begin with discussion of how one would convert a two-dimensional developed layout of a racetrack style winding into a full threedimensional model. Using a similar technique, a model can be created for use in an EM analysis package. The results from this analysis can then be used to apply the resulting loads back onto the geometric model for mechanical analysis.
\end{abstract}

Keywords: electrical windings, modeling, pulsed alternators, EM analysis

\section{INTRODUCTION}

The types of electrical windings described in this paper are utilized in the latest generation pulsed alternator developed by The University of Texas at Austin Center for Electromechanics (UT-CEM). The focus of this paper is to describe the techniques utilized by CEM-UT to accurately model these windings, as well as prepare these models for use in threedimensional electromagnetic (EM) analyses. A large effort was put forth to develop an efficient means of creating these models. As a machine design matures throughout its design phase, it is often necessary to modify geometry of the electrical windings, thus minimizing the time required to implement these changes is essential. The same is true for any electromagnetic analysis models required. Once results are obtained from an EM load analyses, techniques are required to aid in visualizing their magnitude and location. Again, these were developed considering both utility and ease of creation. Finally, methods of transferring these results for further mechanical analysis were needed. Different means were used depending on the particular application.
The modeling methods described in this paper are useful for two different styles of windings. One type is made up of solid conductors cut from a single cylinder. The other is one consisting of stranded conductors which are formed to the appropriate geometry. Due to the large radial and tangential loads seen by the windings in a pulsed alternator, it is imperative that a high fidelity modeling technique be utilized. Often, numerous load cases need to be examined with EM analysis software, and some of these load cases may require detailed mechanical analysis as well. Along with load analyses, these models can also be used to quantify the sensitivity to misalignment of rotor and stator axes, as well as rotor axial position.

\section{GEOMETRIC MODELING}

The major difficulty of modeling racetrack style coils is translating a two-dimensional developed view of the coils into a three-dimensional solid model. However, using CAD (Computer Aided Design) software that allows for automation eases the difficulty of describing a complicated geometry. The modeling performed by UT-CEM was done using a Visual Basic program to control the SolidWorks solid modeler. By using this type of method, the majority of the modeling tasks are performed automatically.

The first step in generating a coil model is constructing a two-dimensional developed view of the coils. Essentially, this is a layout showing how the coils would appear if one were to "unwrap" the coils into a flattened view. This would result in a drawing whose axial dimensions are unchanged, but the dimensions along the circumferential direction is converted to arc lengths at some developed radius. Typically, this is done such that the developed radius is that of the center of the conductor cross section. By laying out the coils in this developed view using only lines and arcs, the three dimensional model can be described mathematically, easing its construction. Fig. 1 shows a set of coils from a four-pole machine laid out in this manner.

This research was sponsored by the U.S. Army Research Laboratory through Lockheed-Martin Missile and Fire Control under contract number 4300050944. 


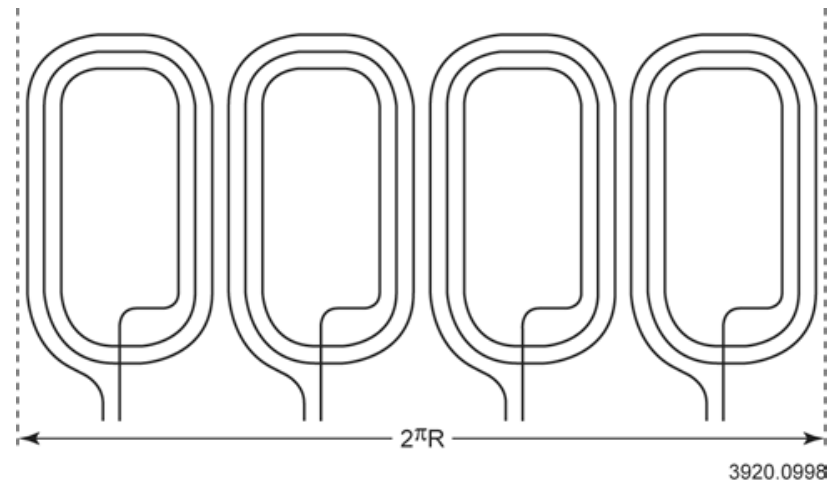

Figure 1. Developed view of electrical windings

While the entire coil is drawn at one developed radius, some conductors may not actually reside at that radial location. This tends to be the case in the end turn sections of the coil where the conductors need to cross over each other, pushing some conductors to higher radial locations. Thus, when deriving the mathematical description of the coil path, this change in radius needs to be accounted for. There are two sets mathematical relations that need to be derived to fully describe the coil path. One for lines lying in a developed view, and the other for arcs in a developed view.

To derive the relation that describes the 3D path of a line given its 2D developed view, the following parameters are needed:

- $\left(\mathrm{X}_{\mathrm{s}}, \mathrm{Y}_{\mathrm{s}}\right)$ - The coordinates of the start point of the line

- $\left(X_{e}, Y_{e}\right)$ - The coordinates of the end point of the line

- $\mathrm{R}_{\mathrm{d}}$ - The radius at which the developed view was created

- $\quad \mathrm{R}_{\mathrm{a}}$ - The actual radius at which the line lies

- $\mathrm{L}$ - Length of the line (Can be calculated with the above coordinates)

The coordinate values are given such that the $\mathrm{Y}$ values correspond to the axial direction and the $\mathrm{X}$ values correspond to the circumferential direction. Given these inputs, a relation can be derived to calculate the path location in space at any distance, $\delta$, along the length of the line. The following is the resulting relationship:

For $0 \leq \delta \geq \mathrm{L}$ :

$$
\begin{aligned}
& X(\delta)=R_{a} \operatorname{Cos}\left(\frac{X_{s}+\frac{\delta}{L}\left(X_{e}-X_{s}\right)}{R_{d}}\right) \\
& Y(\delta)=R_{a} \operatorname{Sin}\left(\frac{X_{s}+\frac{\delta}{L}\left(X_{e}-X_{s}\right)}{R_{d}}\right) \\
& Z(\alpha)=Y_{S}+\frac{\delta}{L}\left(Y_{e}-Y_{s}\right)
\end{aligned}
$$

There are two special cases of the above relations. One is for a line that travels only along the axial direction, such as in the active length section of the coil. The other is a line that travels only circumferentially, never changing axial location. In both cases the relations can be simplified. There is no need to use these simplified relations the actual program calculating the path, but they do serve as a reality check to verify the validity of the relations.

In the case of line that only travels in the axial direction, the $\left(X_{e}-X_{s}\right)$ term equals zero. Therefore the first two equations simplify to a constant value, which yields a line that lies only in one circumferential location as expected. Similarly, for a line that travels only in the circumferential direction, the $\left(\mathrm{Y}_{\mathrm{e}}-\mathrm{Y}_{\mathrm{s}}\right)$ term is zero, making the third equation simplify to a constant. This gives a path that does not change axial location, which is correct.

Similar to the derivation for lines, the relations for an arc need the following parameters from the 2D developed view:

- $\left(X_{c}, Y_{c}\right)$ - Coordinates of the arc center point

- $\alpha_{\mathrm{s}}$ - Starting angle of the arc

- $\alpha_{e}-$ Ending angle of the arc

- $r$ - Radius of the arc

- $\mathrm{R}_{\mathrm{d}}$ - The radius at which the developed view was created

- $\mathrm{R}_{\mathrm{a}}$ - The actual radial location at which the arc lies

Using these values, a relation can be determined which gives the location of any point along the arc as a function of the angle from the starting location. The resulting relations are given below.

For $\alpha \mathrm{s} \leq \alpha \geq \alpha \mathrm{e}$ :

$$
\begin{aligned}
& X(\alpha)=R_{a} \operatorname{Cos}\left(\frac{X_{c}}{R_{d}}+\frac{r}{R_{d}} \operatorname{Cos}(\alpha)\right) \\
& Y(\alpha)=R_{a} \operatorname{Sin}\left(\frac{X_{C}}{R_{d}}+\frac{r}{R_{d}} \operatorname{Sin}(\alpha)\right) \\
& Z(\alpha)=Y_{c}+r \operatorname{Sin}(\alpha)
\end{aligned}
$$

Now that the location of any point on the coil path can be determined, the next step in creating the conductor geometry is creating the cross section of the conductor at these locations. To do this, the plane in which the cross section lies must be determined. To define the plane, it is necessary to take the first derivative of the above functions. This can be used to define a unit vector that is tangent to the path at any point. Another unit vector can be determined that goes directly radially outward from the point on the path. By taking the vector product of the tangent vector and the radial vector, a third vector orthogonal to these two can be determined. The plane of the cross section lies in the plane defined by the radial and orthogonal vectors.

Once the plane of the cross section is known, and given the thickness and width of the conductor, the coordinates defining the corners of the conductor can be calculated. By using the automation features of a CAD package, the cross sections can 
then be constructed along the coil path, without manual input, as often as needed to create accurate model of the coil. The solid model can then be created by lofting from one cross section to the next. It is recommended that separate lofts be created for each separate line or arc from the original 2D developed view. This allows the user to enforce that the straight sections terminate exactly perpendicular to the beginnings of each curved sections. While it would be faster to create a single loft defining the entire coil, surface wrinkling can occur and often there will be local perturbations at the transition between lines and arcs. Fig. 2 shows a solid model of a coil being created in SolidWorks, the CAD packaged used at UT-CEM.

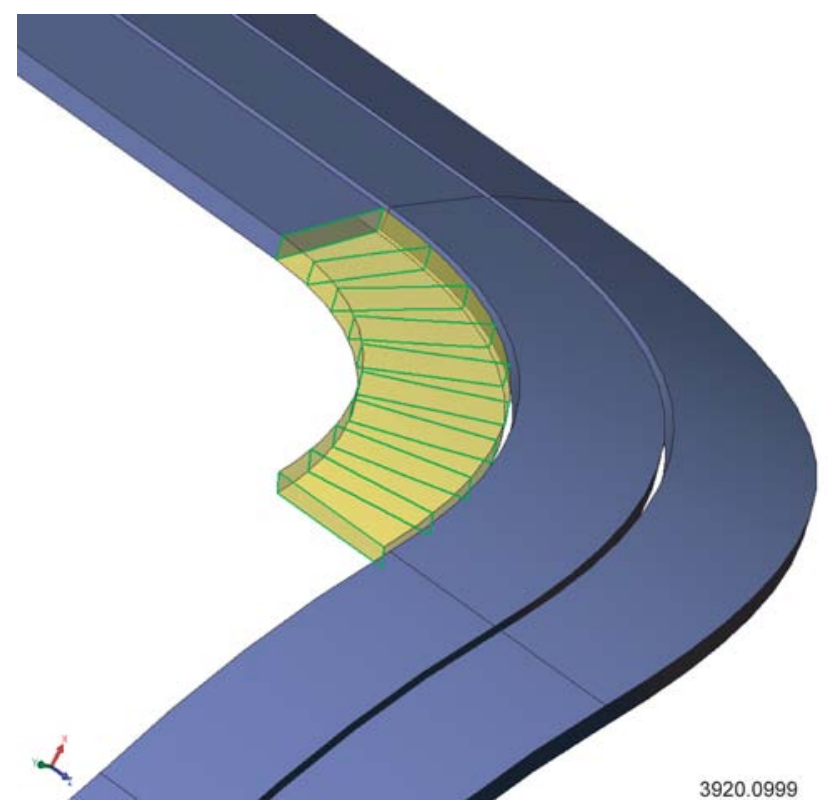

Figure 2. Electrical coil constructed by lofting through cross sections

The above method is appropriate when constructing a model of a coil in which the conductor's cross-section remains rectangular throughout its length. This is true for the case of coils made of a transposed conductor such as litz wire. However, some of the coils that UT-CEM designs are to be water-jet cut or milled out of a solid cylinder. These conductors have a varying cross section. In particular, the conductors are trapezoidal in the active length region of the coil, and transition to a rectangular cross section at the top of an arc in the end-turn region. But, the space between the conductors is a constant width defined by the size of the cutting tool. In these cases, instead of modeling the path of the conductor, the path of the cutting tool was modeled. The cross-sections defined by the cutter width are then used to create lofted cuts from a cylinder. Thus leaving the conductors as the finished model. Fig. 3 shows this operation being performed in SolidWorks. In this type of operation, it is possible that the finished model can actually be used in defining the cutter path in a computer numerically controlled (CNC) machine tool.

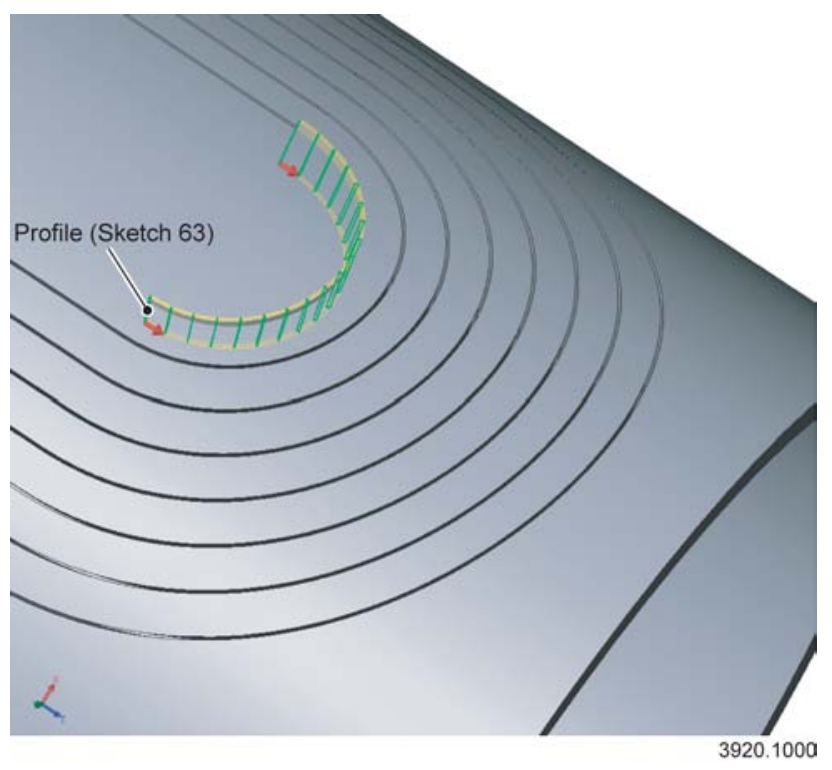

Figure 3. Electrical coil constructed using lofted cuts from a solid cylinder

\section{ELECTROMAGNETIC (EM) ANALYSIS}

In addition to using the previously described method to create a solid model of the electrical windings of a machine, the same technique can be to generate a model for performing electromechanical analyses. Most analysis software packages allow for some sort of data input though a text file. Using the methods described previously, the necessary input file can be created to import the model into the software package. In the case of the work performed at UT-CEM, the EM analysis package used was Opera3D from the Vector Fields Company.

Opera3D allows the user to define source conductors using 8 node brick elements. By using the coordinate values of the conductor cross-section vertices calculated using the previously mentioned method, these elements were calculated programmatically and written into an appropriate text file for import. Once these elements were created and assigned a current density, Opera3D could calculate the resulting EM forces on each element due to the interaction of all the conductors in a machine. These forces could then be used later for visualizing any area of high loads for further inspection.

Another use of the coil geometry is to verify the inductance of the coils. CEM-UT uses an in house code for calculating coil inductances. Previously, this code used idealized coils generated automatically by the code. But through minor modifications, the code was adapted such that it could take 
input generated by the above methods to analyze the coils as they were to be truly constructed.

\section{VISUALIZATION}

Once the EM loads have been determined using Opera3D, the next challenge is visualizing the resulting loads. The two methods found to be the most useful at UT-CEM were colored contour plots and developed stem plots. In both cases, Opera3D did not provide a visualization method, so outside programs were used. In the case of colored contour plots, the conductor geometry and load values were imported into Cosmos/M. By recreating the geometry using the same eight node brick elements, and importing the calculated loads as temperature values, the contour plots could be created. Since Opera3D calculates the net force on each conductor element, and each element does not necessarily have the same volume, the loads had to be first normalized so that the relative magnitude on each element could be compared. This was typically done by dividing the load value by the length of the element, giving units of force/length. Fig. 4 shows an example of a radial load contour plot.

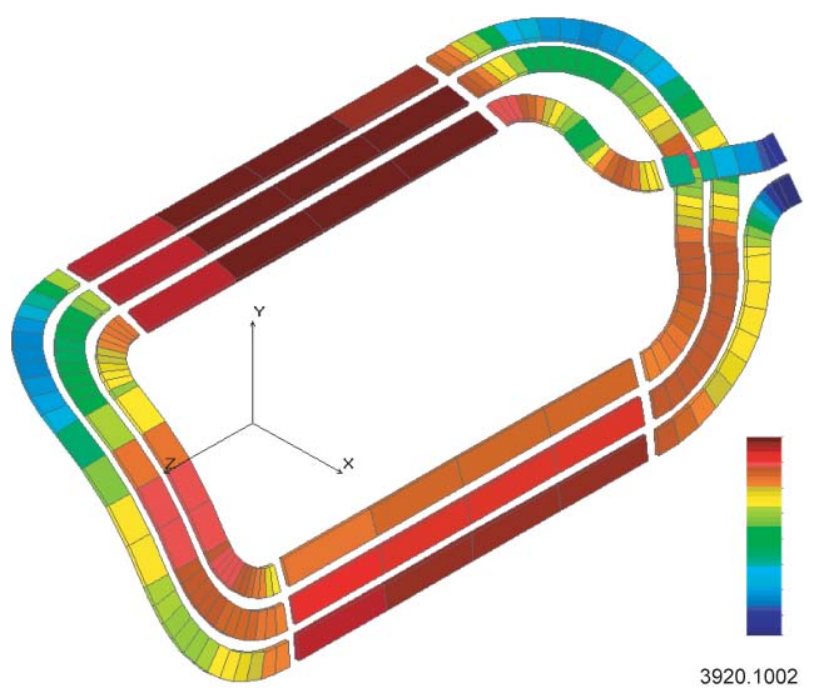

Figure 4. Example radial EM load contour plot

Similar to the contour plots, developed stem plots were also used to visualize load results. In this case, the plots were created using MatLab. Once again, the loads were normalized in the same fashion and the conductor geometry was loaded in using a text file formatted for input to automate the process. Then using a small MatLab routine, the stem plots could be created. A sample stem plot can be seen in Fig. 5.

\section{MECHANICAL ANALYSIS}

After EM results have been calculated and visualized for multiple load cases, the cases that require further inspection need to be prepared for mechanical analysis. Different methods are required, depending on the type of analysis to be performed. In some cases, the loads might need to be transferred for use in a full three-dimensional model that includes the true coil geometry in the model. To ease the transfer of the EM load data, the mesh used in the mechanical model needs to match that of the mesh used in the EM model. The geometry creation program was written to allow the user to set the number of segments into which the conductor will be divided. So, a compromise can be made such that the number of elements is enough to provide adequate fidelity in the mechanical model without making the number too large to efficiently run the EM analysis. Having matching meshes allows for the transfer of the EM loads to the mechanical model as body forces on the elements.

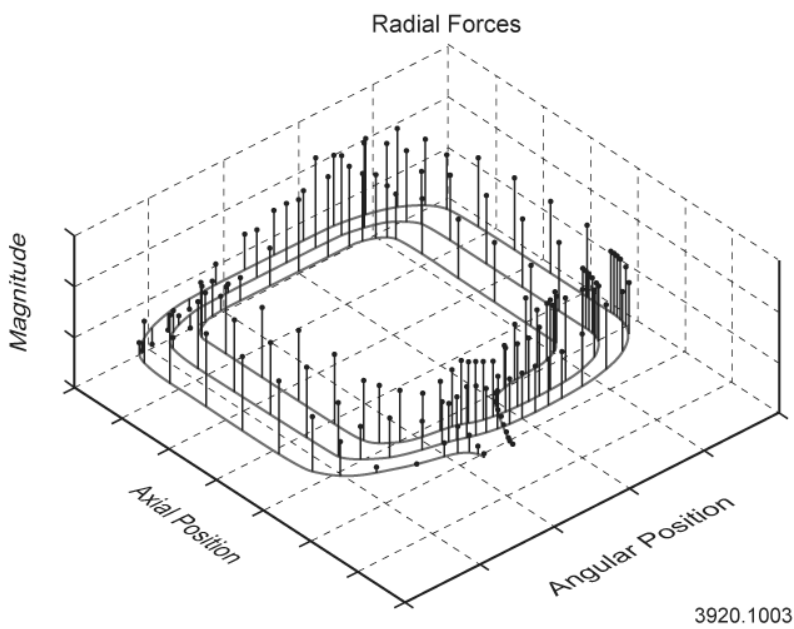

Figure 5. Example radial EM load stem plot

A different method may be required for a model that does not directly contain the winding geometry. For instance, UTCEM created a mechanical model of a stator housing and needed account for the loads developed in the windings. Thus a means of transferring the loads to inner bore of the stator housing was needed. The mesh on the bore of the stator tube was divided into equal angular divisions, but was divided along the axis in a non-uniform manner. To accomplish this, the conductor elements were sub-divided into small units. Based off the angular and axial location of the centers of these subelements, a percentage of the total force on the element was added to the force seen by the corresponding element on the stator housing mesh. It was found that to create a smooth distribution across the elements it was necessary to divide the conductor elements into quite small units, but as no actual analysis was being performed, this was not a time intensive task. The same program used for the other post processing tasks was once again utilized to perform this task. Fig. 6 is a surface contour map showing a typical load distribution to be applied to the inner surface of the stator housing. 


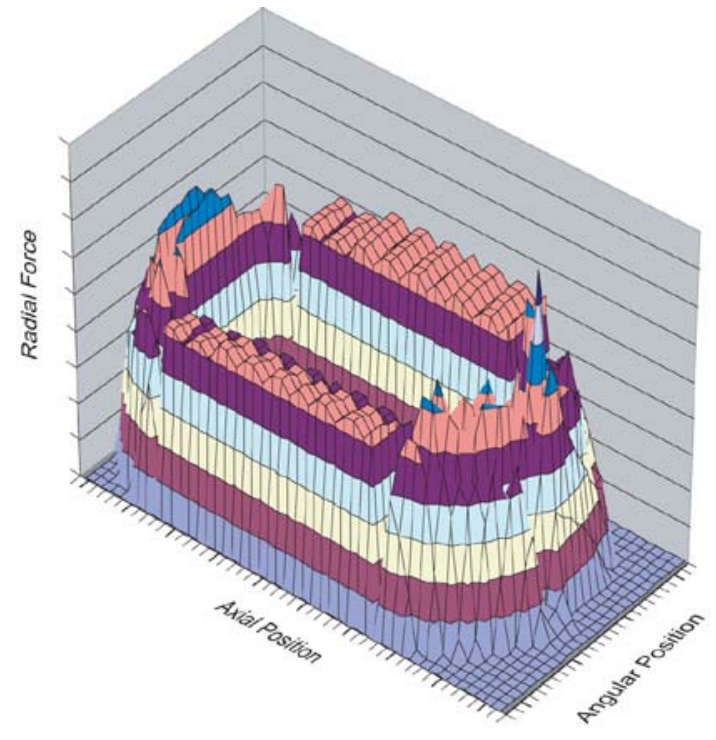

3920.1004

Figure 6. Example surface contour map of radial loads

\section{CONCLUSION}

UT-CEM has developed a set of routines for performing and automating many of the necessary tasks involved in the design and analysis of electrical windings for pulsed alternators. These techniques begin with the construction of a solid model of the windings starting with a two-dimensional developed view. This includes a means of producing constant cross-section conductors such a litz wire as well as varying cross-section conductors such a those produced by cutting from a solid cylinder. These same techniques were then modified to aid in the construction of models for use in EM analyses such as force and inductance calculations. In the case of force studies, the routines were expanded to facilitate the creation of different visualization methods. In order for mechanical analyses to be performed using the loads calculated in the EM models, different means were used in transferring the data to the mechanical models. All these tools will no doubt decrease the time required for studying future pulsed alternator designs.

\section{REFERENCES}

[1] SolidWorks (2003) [Computer Program] SolidWorks Corporation, Concord, MA

[2] Opera3D (9.0) [Computer Program] Vector Fields Corporation, Aurora, IL

[3] Visual Basic (6.0) [Computer Program] Microsoft Corporation, Redmond, WA 